\title{
QUALIDADE DE VIDA EM VOZ: \\ ESTUDO NA DOENÇA DE PARKINSON IDIOPÁTICA \\ E NA DISFONIA ESPASMÓDICA ADUTORA
}

\section{Quality of life in voice: a study in Parkinson's disease and in adductor spasmodic dysphonia}

\author{
Bárbara Pereira Lopes (1), Riviana Rodrigues das Graças ${ }^{(2)}$, lara Barreto Bassi (3), \\ Antônio Lobo de Rezende Neto ${ }^{(4)}$, João Batista de Oliveira ${ }^{(5)}$, \\ Francisco Eduardo Costa Cardoso ${ }^{(6)}$, Ana Cristina Côrtes Gama ${ }^{(7)}$
}

\begin{abstract}
RESUMO
Objetivo: avaliar o impacto causado pela alteração vocal na qualidade de vida (QV) dos pacientes com doença de Parkinson (DP) idiopática e com disfonia espasmódica adutora (DEA). Método: 0 estudo foi realizado com 56 indivíduos do sexo feminino, dos quais 28 compunham o grupo controle; 14, o grupo de DEA, no período anterior ao tratamento com toxina botulínica; e 14, o grupo de DP idiopática. Os participantes preencheram o questionário de Qualidade de Vida em Voz (QVV) validado para o português brasileiro. Para verificar a diferença entre as médias dos grupos foi utilizado o método de análise de variância por postos de Kruskal-Wallis e o teste de Tamhane para comparações múltiplas, com significância $<0,05$. Resultados: as médias de idade dos grupos foram 66,18 para 0 grupo controle, 67,21 DP e 59,7 DEA, não havendo diferença estatística entre as médias. No protocolo QVV as médias dos domínios sócio-emocional, físico e escore total foram maiores para o grupo controle, seguido pelo grupo de DP idiopática e por fim o grupo da DEA, sendo a diferença entre elas estatisticamente significante. Além disso, houve diferença estatística para cada par de grupos, referente aos domínios do protocolo QVV, exceto no que se refere ao par controle e DP idiopática, no domínio sócio emocional $(\mathrm{p}=0,076)$. Conclusão: o maior impacto negativo na $\mathrm{QV}$ relacionado à voz está nos indivíduos com DEA, seguido por aqueles com DP idiopática e por fim os indivíduos do grupo controle.
\end{abstract}

DESCRITORES: Doença de Parkinson; Distonia; Disartria; Voz; Distúrbios da Voz; Qualidade de Vida

(1) Aluna do curso de Graduação em Fonoaudiologia da Universidade Federal de Minas Gerais - UFMG, Belo Horizonte, MG, Brasil.

(2) Fonoaudióloga graduada pelo Curso de Fonoaudiologia da Universidade Federal de Minas Gerais - UFMG, Belo Horizonte, MG, Brasil.

(3) Fonoaudióloga; Doutoranda em Saúde Pública pela Universidade Federal de Minas Gerais - UFMG, Belo Horizonte, MG, Brasil.

(4) Otorrinolaringologista da clínica Núcleo de Otorrino de BH, Belo Horizonte, MG, Brasil; Mestre em ciências da Saúde pela Universidade Federal de Minas Gerais - UFMG, Belo Horizonte, MG, Brasil.

(5) Otorrinolaringologista da clínica Núcleo de Otorrino de $\mathrm{BH}$, Belo Horizonte, MG, Brasil; Mestre em Cirurgia pela Universidade Federal de Minas Gerais - UFMG, Belo Horizonte, MG, Brasil.

\section{INTRODUÇÃO}

Aos prejuízos de fala e voz consecutivos a lesões periféricas e centrais do sistema nervoso, dá-se o nome de disartria ${ }^{1}$. Muitas são as subdivisões

(6) Neurologista; Professor Titular da Faculdade de Medicina da Universidade Federal de Minas Gerais - UFMG, Belo Horizonte, MG, Brasil; Doutor em Biologia Celular pela Universidade Federal de Minas Gerais - UFMG, Belo Horizonte, MG, Brasil.

(7) Fonoaudióloga; Professora associada do departamento de fonoaudiologia da Universidade Federal de Minas Gerais UFMG, Belo Horizonte, MG, Brasil; Doutora em Ciências dos Distúrbios da Comunicação Humana pela Universidade Federal de São Paulo, Brasil.

Conflito de interesses: inexistente 
derivadas desse termo, mas o presente estudo se aterá a apenas duas delas: as disartrias hipocinéticas e hipercinéticas.

Disartrias hipocinéticas são caracterizadas pela presença de rigidez e extensão reduzida de movimentos, além da dificuldade em iniciá-los e o tremor de repouso. Tal alteração está presente na doença de Parkinson (DP), conceituada na literatura como uma doença de etiologia idiopática que afeta a produção de dopamina, neurotransmissor sintetizado na substância negra do mesencéfalo, devido à redução da quantidade de neurônios nessa região cerebral ${ }^{2,3}$.

$\mathrm{Na}$ fase inicial da doença, as alterações de voz e articulação não são suficientes para causar grandes prejuízos na comunicação oral. Entretanto, à medida que a doença avança em gravidade o impacto negativo na comunicação também se eleva a ponto de tornar-se altamente prejudicada ${ }^{4}$. Intensidade vocal reduzida, qualidade vocal rouca, soprosa, instabilidade fonatória, articulação reduzida e imprecisa e graus variados de redução da inteligibilidade compõem o quadro de alterações vocais e de fala em parkinsonianos ${ }^{2}$.

Por sua vez, as disartrias hipercinéticas envolvem movimentos involuntários, acelerados e descontrolados $^{2}$. A exemplo tem-se a Disfonia Espasmódica Adutora (DEA) ou também denominada Distonia Focal Laríngea de origem central, classificada como um distúrbio vocal, que afeta predominantemente mulheres e é caracterizado por espasmos laríngeos em fechamento, que são contrações intermitentes e involuntárias dos músculos laríngeos. Como resultado, é possível identificar uma voz irregular, quebrada, tensa e estrangulada ${ }^{5}$.

Enquanto na DP idiopática ocorre a diminuição da iniciativa motora causada pela redução da estimulação cortical exercida pelas projeções tálamo-corticais, na DEA encontra-se o surgimento de movimentos anormais pela redução da inibição do tálamo e hiperatividade da alça tálamo-cortical ${ }^{6}$. Estudos realizados com indivíduos parkinsonianos demonstraram que as alterações vocais mais frequentes estavam relacionadas à qualidade da voz, em específico soprosidade e rouquidão; ao padrão articulatório impreciso e à redução da intensidade vocal $4,7,8$. Em contrapartida, a DEA apresenta um quadro vocal caracterizado por uma voz tensa, estrangulada e frequentemente interrompida por pausas ${ }^{9}$. Dessa forma, ao se considerar que a DP idiopática e a DEA possuem bases neurológicas diferentes, o que leva à manifestação de sinais vocais também distintos, acredita-se que o impacto negativo na Qualidade de Vida $(\mathrm{QV})$ relacionada à voz desses sujeitos será influenciado pelo grau de comprometimento vocal provocado pela doença.

Devido a todas as alterações supracitadas, estudos vêm sendo feitos na tentativa de se conhecer o impacto que a DP e a DEA ocasionam na QV destes indivíduos. Entretanto, além do reduzido número de estudos que exploram este assunto, notou-se que nenhum deles analisa, em específico, o impacto da DP e da DEA na qualidade de vida em voz desses sujeitos, entendendo que o impacto na comunicação em ambos os grupos é distinto, como se observa na literatura ${ }^{2,4,5}$.

Dessa forma, o objetivo do presente estudo consiste em avaliar o impacto causado pela alteração vocal na QV dos pacientes com doença de Parkinson idiopática e na Disfonia Espasmódica de Adução.

\section{MÉTODO}

O presente estudo é uma pesquisa de corte transversal da qual participaram 56 indivíduos do sexo feminino, sendo 14 pacientes, com idade média de 59 anos, atendidas na clínica Núcleo de Otorrino de $\mathrm{BH}$ com o diagnóstico de Disfonia Espasmódica Adutora em período anterior ao tratamento com toxina botulínica; e 14 pacientes, também do sexo feminino, com idade média de 67 anos, com o diagnóstico Doença de Parkinson Idiopática segundo os critérios do Banco de Cérebros da Sociedade de Parkinson do Reino Unido e em tratamento na Clínica de Distúrbios do Movimento, Serviço de Neurologia do Hospital Bias Fortes do HC/UFMG, além disso, as participantes deveriam estar classificadas entre os estágios e 1 a 3 da escala de estágios de incapacidade de Hoehn e Yahr. Para compor o grupo controle, convidou-se 28 acompanhantes de pacientes assistidos pelo HC/UFMG, com idade média de 66 anos, a fim de que os grupos fossem pareados por sexo e contexto sócio-cultural. Foram critérios de inclusão para este grupo a ausência de distúrbio da comunicação e de histórico de afecções neurológicas.

Foram excluídos da pesquisa os indivíduos do sexo masculino, que fizeram fonoterapia, aqueles que não compreenderam a tarefa solicitada e paciente que já haviam se submetido à injeção de toxina botulínica nos músculos laríngeos, sendo este último critério aplicado aos casos de disfonia espasmódica.

Para a análise da qualidade de vida foi utilizado o questionário de Qualidade de Vida em Voz (QVV), validado para o português por brasileiro ${ }^{10}$. É um instrumento que tem como finalidade medir o impacto de uma disfonia em diversos aspectos 
da vida relacionados à comunicação oral, sendo composto por 10 itens separados em dois domínios: sócio-emocional, contemplado pelos itens 4,5,8 e10; e físico, contemplado pelos itens 1,2,3,6,7 e 9. O questionário segue uma escala numérica de 1 a 5 sendo que os valores da escala correspondem às seguintes situações: $1=$ não é um problema, $2=$ é um problema pequeno, $3=$ é um problema moderado/médio, 4= é um grande problema e $5=$ é um problema muito grande. Além do cálculo dos escores de cada domínio, também se calcula o escore total. Quanto mais próximo de 100 for o resultado, melhor será a qualidade de vida relacionada à voz do sujeito (Figura 1).

Todas as participantes assinaram o Termo de Consentimento Livre e Esclarecido e esta pesquisa foi aprovada pelo Comitê de Ética em Pesquisa da UFMG - COEP (ETIC 025/04 e 676/07).

A análise estatística dos dados foi realizada por meio do programa estatístico SPSS (Statistical Package for the Social Sciences) versão 17.0. Primeiramente foi realizada uma análise descritiva dos dados com medidas de tendência central e dispersão. Para verificar a diferença entre as médias dos grupos foi utilizado o método de análise de variância por postos de Kruskal-Wallis e o teste de Tamhane para comparações múltiplas. Os resultados considerados significantes foram aqueles com $p \leq 0,05$.

\section{RESULTADOS}

Participaram do estudo um total de 56 sujeitos, sendo 28 do grupo controle com média de idade de 66 anos, 14 do grupo com Doença de Parkinson com média de idade de 67 anos e 14 sujeitos com disfonia espasmódica com média de idade de 59 anos. Não foi observada diferença estatística entre as médias de idade dos grupos (Tabela 1).

Analisando a Figura 2 é possível observar que as médias dos domínios sócio-emocional, físico e escore total são maiores para o grupo controle, seguida pelo grupo de doença de Parkinson e por fim o grupo da disfonia espasmódica adutora.

$\mathrm{Na}$ Tabela 2 observa-se que houve diferença entre as médias nos três domínios do protocolo de QV nos grupos estudados.

$\mathrm{Na}$ análise dos domínios do protocolo de QV para cada par de grupos, apenas na comparação entre o par controle e DP no domínio sócio-emocional, não houve diferença estatisticamente significante (Tabela 3). 
Nome

Data:

Idade:

Estamos procurando compreender melhor como um problema de voz pode interferir nas atividades de vida diária. Apresentamos uma lista de possíveis problemas relacionados à voz. Por favor, responda a todas as questões baseadas em como sua voz tem estado nas duas últimas semanas. Não existem respostas certas ou erradas.

Para responder ao questionário, considere tanto a gravidade do problema, como sua frequência de aparecimento, avaliando cada item abaixo de acordo o tamanho do problema que você tem. A escala que você irá utilizar é a seguinte:

1 = não é um problema

2 = é um problema pequeno

3 = é um problema moderado/médio

4 = é um grande problema

5 = é um problema muito grande

Por causa de minha voz,

O quanto isto é um problema?

1. Tenho dificuldades em falar forte (alto) ou ser ouvido em lugares barulhentos.

2. O ar acaba rápido e preciso respirar muitas vezes enquanto eu falo.

$\begin{array}{lllll}1 & 2 & 3 & 4 & 5\end{array}$

3. Às vezes, quando começo a falar não sei como minha voz vai sair.

$\begin{array}{lllll}1 & 2 & 3 & 4 & 5\end{array}$

4. Às vezes, fico ansioso ou frustrado (por causa da minha voz).

$\begin{array}{lllll}1 & 2 & 3 & 4 & 5\end{array}$

5. Às vezes, fico deprimido(por causa da minha voz).

$\begin{array}{lllll}1 & 2 & 3 & 4 & 5\end{array}$

6. Tenho dificuldades em falar ao telefone (por causa da minha voz).

$\begin{array}{lllll}1 & 2 & 3 & 4 & 5\end{array}$

7. Tenho problemas no meu trabalho ou para desenvolver minha profissão (por causa da minha voz).

$\begin{array}{lllll}1 & 2 & 3 & 4 & 5\end{array}$

8. Evito sair socialmente (por causa da minha voz).

$\begin{array}{lllll}1 & 2 & 3 & 4 & 5\end{array}$

9. Tenho que repetir o que falo para ser compreendido.

$\begin{array}{lllll}1 & 2 & 3 & 4 & 5\end{array}$

10. Tenho me tornado menos expansivo (por causa da minha voz).

123

5

Figura 1 - Protocolo de Qualidade de Vida em Voz - QVV (Gasparini, Behlau, 2006) 
Tabela 1 - Comparação das médias de idade entre os grupos Controle, Parkinson e Espasmódica

\begin{tabular}{llcccccc}
\hline & N & Média & $\begin{array}{c}\text { Desvio } \\
\text { Padrão }\end{array}$ & Mínimo & Máximo & $p$ \\
\hline \multirow{3}{*}{ Idade } & Controle & 28 & 66,18 & 10,09 & 41 & 89 & \\
& Parkinson & 14 & 67,21 & 10,86 & 52 & 90 & 0,096 \\
& Espasmódica & 14 & 59,07 & 12,81 & 33 & 74 & \\
\hline
\end{tabular}

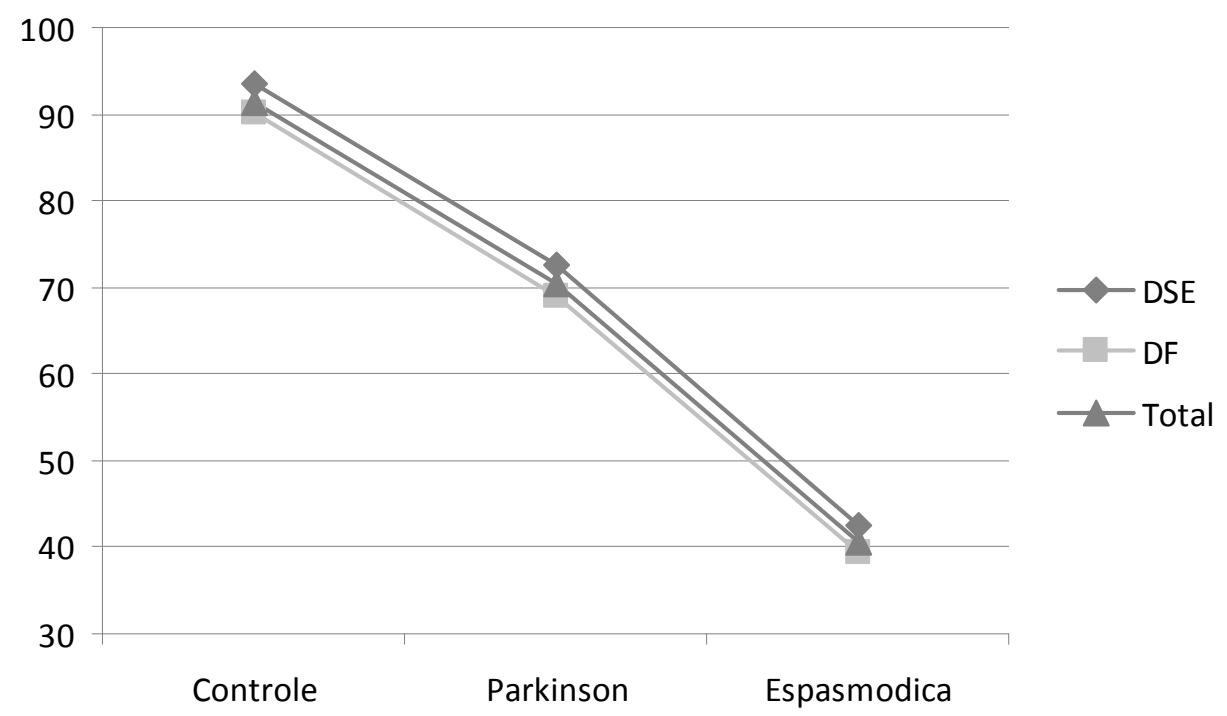

DSE= domínio sócio-emocional $\mathrm{DF}=$ domínio físico

Figura 2 - Representação gráfica das médias dos grupos para os domínios do QVV

Tabela 2 - Distribuição de média, desvio padrão, mínimo e máximo para cada grupo

\begin{tabular}{llcccccc}
\hline & N & Média & $\begin{array}{c}\text { Desvio } \\
\text { Padrão }\end{array}$ & Mínimo & Máximo & $\boldsymbol{p}$ \\
\hline \multirow{3}{*}{ DSE } & Controle & 28 & 93,53 & 9,69 & 68,75 & 100,00 & \\
& Parkinson & 14 & 72,54 & 30,84 & 12,50 & 100,00 & $0,000^{*}$ \\
& Espasmódica & 14 & 42,41 & 30,54 & 0,00 & 93,75 & \\
\hline \multirow{2}{*}{ DF } & Controle & 28 & 90,03 & 11,12 & 54,20 & 100,00 & \\
& Parkinson & 14 & 69,05 & 26,30 & 8,30 & 100,00 & $0,000^{*}$ \\
& Espasmódica & 14 & 39,30 & 24,45 & 4,20 & 79,20 & \\
\multirow{2}{*}{ Total } & Controle & 28 & 91,43 & 9,51 & 65,00 & 100,00 & \\
& Parkinson & 14 & 70,36 & 26,85 & 10,00 & 100,00 & $0,000^{*}$ \\
& Espasmódica & 14 & 40,54 & 25,29 & 12,50 & 85,00 & \\
\hline
\end{tabular}

Teste Kruskal-Wallis

DSE= domínio sócio-emocional

$\mathrm{DF}=$ domínio físico

${ }^{*}=$ valor de $p$ estatisticamente significante. 
Tabela 3 - Comparação de médias entre os grupos Controle, Parkinson e Espasmódica

\begin{tabular}{lllccc}
\hline \multirow{2}{*}{ Domínios do QVV } & & \multirow{2}{*}{ Grupos } & $\boldsymbol{p}$ & \multicolumn{2}{c}{$95 \%$ Intervalo de confiança } \\
\cline { 5 - 6 } & & & & Inferior & Superior \\
\hline \multirow{2}{*}{ DSE } & Controle & Parkinson &, 076 & $-1,82$ & 43,80 \\
& Controle & Espasmódica &, $000^{*}$ & 28,52 & 73,71 \\
& Parkinson & Espasmódica &, $045^{*}$ & 0,53 & 59,73 \\
\hline \multirow{2}{*}{ DF } & Controle & Parkinson &, $035^{*}$ & 1,34 & 40,63 \\
& Controle & Espasmódica &, $000^{*}$ & 32,41 & 69,05 \\
& Parkinson & Espasmódica &, $014^{*}$ & 5,25 & 54,25 \\
\hline \multirow{2}{*}{ Total } & Controle & Parkinson &, $037^{*}$ & 1,15 & 40,99 \\
& Controle & Espasmódica &, $000^{*}$ & 32,09 & 69,69 \\
& Parkinson & Espasmódica &, $017^{*}$ & 4,66 & 54,98 \\
\hline
\end{tabular}

Teste de comparação múltipla Tamhane

DSE= domínio sócio-emocional

$\mathrm{DF}=$ domínio físico

${ }^{*}=$ valor de $p$ estatisticamente significante.

\section{DISCUSSÃO}

Nos últimos anos a QV tem sido alvo dos pesquisadores e terapeutas, por fornecer dados importantes sobre a perspectiva do paciente quanto ao seu estado atual, e muitos são os instrumentos utilizados em sua mensuração. Os protocolos de análise da QV podem ser genéricos, ou seja, aplicáveis a maior parte da população, ou específicos, que podem estar relacionados à idade ou mesmo ao comprometimento apresentado pelo sujeito ${ }^{11-13}$.

O QVV é um instrumento específico que avalia a qualidade de vida em pacientes disfônicos e tem sido amplamente utilizado na clínica fonoaudiológica $^{10}$. Dessa forma, pacientes com DEA ou DP, por possuírem comprometimento comunicativo, tendem a apresentar impactos mais negativos na qualidade de vida, quando comparados àqueles indivíduos que não possuem alterações comunicativas ${ }^{14-16}$. Dados semelhantes foram encontrados no presente estudo, no qual se verificou um maior impacto negativo na QV do grupo DEA, seguido do grupo DP, e por fim, o grupo controle (Figura 2).

Houve diferença entre as médias nos três domínios do protocolo de QV nos grupos estudados (Tabela 2), com escores mais elevados no grupo controle. Em estudo realizado ${ }^{15} \mathrm{com} 103$ mulheres com idade igual ou superior a 60 anos, observou-se que $93,21 \%$ das idosas apresentaram um escore total entre 70 e 100, bem como, um outro estudo 17, também realizados com idosos, verificou que a qualidade vocal destes não interferiam na sua comunicação, o que confirmam os elevados escores apresentados pelo grupo controle deste estudo. $\mathrm{O}$ grupo DP apresentou escores com valores intermediários, o que representa um significante impacto negativo na QV relacionada a voz, corroborando com a literatura que apresenta a comunicação como influente na $Q V$ de pacientes com DP ${ }^{18}$. Por sua vez, a DEA apresentou piores escores que os demais grupos o que demonstra um grande comprometimento da QV devido a alteração vocal, sendo que resultados semelhantes podem ser encontrados na literatura ${ }^{14,19}$. Dessa forma, entende-se que a QV se relaciona com o comprometimento comunicativo apresentado pelo individuo e por isso o impacto mais negativo na QV do grupo DEA pode estar associado ao maior comprometimento comunicativo neste grupo.

Apesar desta pesquisa não encontrar diferença estatisticamente significante entre o par controle e DP referente ao domínio sócio-emocional (Tabela 3), estudos demonstram que ansiedade, depressão, psicose, apatia dentre outros transtornos, são frequentemente encontrados em pacientes parkinsonianos podendo impactar negativamente sua QV ${ }^{20,21}$. Já nos demais pares de grupos, foi possível verificar que o maior impacto negativo na $Q V$ se deu no grupo DEA, seguido da DP e por fim, o grupo controle. Acredita-se que este fato pode estar relacionado com a distinção estabelecida nos mecanismos fisiológicos que ocorrem em cada doença e a consequente alteração da qualidade vocal que se instala nos sujeitos. Enquanto a voz do paciente pakinsoniano sugere fraqueza, cansaço, timidez e desinteresse por suas características 
de soprosidade, rouquidão, baixa intensidade e imprecisão articulatória, respectivamente; a voz tensa-estrangulada, característica da disfonia espasmódica, transmite ao ouvinte a sensação de desespero, aflição, angústia e falta de ar levando-o muitas vezes a interferir no discurso do falante solicitando que este relaxe ${ }^{22}$. A interrupção do falante com disfonia espasmódica por seu ouvinte pode influenciar na percepção que aquele tem dos prejuízos vocais provocados pela doença e dessa forma levá-lo a apresentar escores mais reduzidos da QV relacionada à voz. Portanto, o presente estudo evidencia que o impacto na QV dos sujeitos é altamente influenciado pelo grau de comprometimento que a afecção proporciona. Indivíduos com maior comprometimento vocal apresentarão um elevado impacto negativo na QV relacionada à comunicação.

$\mathrm{O}$ efeito cada vez mais negativo na $\mathrm{QV}$ à medida em que se eleva a gravidade da disfonia tem sido comprovado pela literatura nacional e internacional 15,23; entretanto, a maioria destes estudos são realizados com disfonia de base comportamental. Ao se referir à disfonia de base neurológica, deve-se levar em consideração que não apenas a voz será comprometida, mas a comunicação como um todo, e dessa forma, a relação direta entre disfonia e QV pode não se aplicar a estes casos.

Uma pesquisa ${ }^{18} \mathrm{com} 7341$ indivíduos na cidade de Madrid, Espanha, observou que algumas doenças crônicas desempenham um maior impacto negativo na QV que outras, fato que se faz ainda mais evidente quando se leva em consideração a análise, de forma separada, dos domínios contidos no instrumento utilizado na mensuração. O domínio relacionado à "dor", por exemplo, sofre um maior impacto em doenças reumáticas e traumatológicas quando comparadas à esquizofrenia e outros transtornos psiquiátricos, enquanto a "aptidão física", por sua vez, é mais influenciada negativamente por doenças crônicas como depressão, esquizofrenia e doença de Alzheimer. Da mesma forma, os autores observaram que as afecções que mais influenciam as "atividades diárias" compreendem a doença de Alzheimer ou de demência, doença de Parkinson e AVCs, bem como apresentou a doença de Parkinson como uma afecção crônica que estabelece um elevado impacto negativo sobre vários dos domínios relacionados à $\mathrm{QV}$, o que concorda com o presente estudo no qual, apesar de ter-se analisado apenas domínios relacionados à comunicação, todos foram influenciados negativamente pela doença.

Apesar do impacto na QV geral ser mais negativo na DP pelo fato desta doença gerar maiores restrições nas atividades de vida diária e mobilidade $^{24}$, o impacto mais negativo, nesta pesquisa, da QV foi no grupo DEA, mostrando que o QVV é um instrumento sensível para analisar a $Q V$ relacionada especificamente à voz.

Cabe ressaltar que este estudo analisou pacientes com disfonia espasmódica de adução em período anterior à injeção de toxina botulínica, o que pode ter exercido significante influência sobre o achado já que estudos comprovam a melhora na QV com este tipo de tratamento ${ }^{14,19,25}$. Além disso, os pacientes parkinsonianos tratados com levodopa podem apresentar melhora em alguns parâmetros vocais, o que pode elevar a QV ${ }^{26}$. Entretanto, a dificuldade em se encontrar pacientes com Parkinson em período anterior ao tratamento medicamentoso fez com que nossa amostra fosse composta por indivíduos sob medicação.

Tratando-se da restrição comunicativa, a diferença estabelecida entre as doenças estudadas evidenciou que o QVV é um questionário de grande utilidade na medição de quão impactante esta restrição se faz na QV . Além disso, a relação estabelecida entre o tipo de disfunção e sua influência na QV dos indivíduos portadores de Doença de Parkinson Idiopática e Disfonia Espasmódica Adutora, leva-nos a refletir a respeito da necessidade de implantação de novas abordagens terapêuticas que sejam capazes de assistir essas populações não apenas no que se refere à doença propriamente dita, mas que proporcionem melhora na Qualidade de Vida relacionada à expressão vocal, estabelecendo uma comunicação mais eficiente. Portanto, a presença de um fonoaudiólogo no tratamento dos sujeitos acometidos por essas afecções faz-se imprescindível, já que este profissional tem como principal função aperfeiçoar as relações comunicativas que por vezes, encontram-se comprometidas em certas patologias neurológicas ${ }^{27}$.

Os resultados da presente pesquisa demonstram que o QVV mostrou ser um instrumento sensível para avaliar o impacto da voz na qualidade de vida de pacientes disártricos.

\section{CONCLUSÃO}

O impacto negativo na QV relacionada à alteração vocal é maior na disfonia espasmódica adutora, seguida da doença de Parkinson idiopática, e por fim os melhores scores são do grupo controle.

\section{AGRADECIMENTOS}

Ao Conselho Nacional de Desenvolvimento Científico e Tecnológico (CNPQ) pelo apoio financeiro. 


\section{ABSTRACT}

Purpose: to evaluate the impact of voice disorders on quality of life of patients with idiopathic Parkinson's disease and with adductor spasmodic dysphonia. Method: the study consisted of 56 females, 28 in the control group; 14, the adductor spasmodic dysphonia group in the period prior to treatment with botulinum toxin; and 14, the group of idiopathic Parkinson's disease. The participants filled in the Voice-Related Quality of life (V-RQOL) questionnaire validated for Brazilian Portuguese. To check the difference between averages of the groups it was used the method of analysis of variance by Kruskal-Wallis and Tamhane test for multiple comparisons, with significance $<0.05$. Results: the average age of the groups were 66.18 for the control group, 67.21 for the Parkinson's disease group and 59.7 for the adductor spasmodic dysphonia group, with no statistical difference between the groups. In the V-RQOL protocol the mean domain social-emotional, physical functioning and total score were higher in the control group, followed by group of Parkinson's disease and, finally, the group of adductor spasmodic dysphonia with statistically significant difference between them. In addition, there was statistical difference for each pair of groups, concerning the areas V-RQOL protocol, except as regards the pair control and Parkinson's disease group in the social-emotional domain $(p=0,076)$ Conclusion: the negative impact of voice on quality of life is greatest in the adductor spasmodic dysphonia group, followed by Parkinson's disease group and at last, the control group.

KEYWORDS: Parkinson Disease; Dystonia; Dysarthria; Voice; Voice Disorders; Quality of Life

\section{REFERÊNCIAS}

1. Le Huche F, Allali A. A Voz: Patologia vocal de origem orgânica. Porto Alegre: Artmed; 2005.

2. Behlau M. Voz: o livro do especialista. Volume 2. Rio de Janeiro. Revinter, 2005.

3. Teixeira Jr AL, Cardoso F. Tratamento inicial da doença de Parkinson. Rev de Neurociênc. jul/set, 2004; 12(3):141-6.

4. Dias AE, Limongi JCP. Tratamento dos distúrbios da voz na Doença de Parkinson: o método Lee Silverman. Arq Neuropsiquiatr. 2003; 61 (1): 61-6.

5. Imamura R, Tsuji DH. Disfonia espasmódica de adução, tremor vocal e disfonia de tensão muscular: é possível fazer o diagnóstico diferencial? [editorial]. Rev Bras. Otorrinolaringol. Jul-agos, 2006; 72(4): 434.

6. Barbosa ER, Haddad MS, Gonçalves MRR. Distúrbios do movimento. In: Nitrine R. Bacheschi LA. A neurologia que todo médico deve saber. $2^{\mathrm{a}}$ ed. São Paulo: Atheneu, 2005. 297-322.

7. Ferreira FV, Cielo CA, Trevisan ME. Força muscular respiratória, postura corporal, intensidade vocal e tempos máximos de fonação na doença de Parkinson. Rev. CEFAC, in press 2010.

8. Ferreira FV, Cielo CA, Trevisan ME. Aspectos respiratórios posturais e vocais da doença de Parkinson: considerações teóricas. Rev. CEFAC. 2011 Mai-jun; 13(3): 534-40.

9. Rowland LA. Merritt: Tratado de neurologia. $2^{\text {a }}$ ed. Rio de Janeiro: Guanabara Koogan, 2007.
10. Gasparini G, Behlau M. Quality of Life: validation of the Brasilian version of the Voice-Related Quality Of Life (V-RQOL) measure. J. Voice. 2009; 23(1): 76-81.

11. Lima MJB, Portela MC. Elaboração e avaliação da confiabilidade de um instrumento para medição da qualidade de vida relacionada à saúde de idosos independentes. Cad Saúde Pública. Ago, 2010; 26(8):1651-62.

12. Ferreira LN, Brito U, Ferreira PL. Qualidade de vida em doentes com asma. Rev Port Pneumol. jan, 2010: 16(1): 23-55.

13. Kasama ST, Brasolloto AG. Vocal perception and quality of life. Pró-Fono R Atual Cient. jan.-abr. 2007; 19 (1): 19-28.

14. Paniello MDRC, Barlow MSWJ, Serna BSJS. Longitudinal follow-up of spasmodic dysphonia. Laryngoscope. Mar, 2008; 118: 564-8.

15. Gama ACC, Alves CFT, Cerceau JSB, Teixeira LC. Correlação entre dados perceptivo-auditivos e qualidade de vida em voz de idosas. Pró-Fono $\mathrm{R}$ Atual Cient. abr-jun, 2009; 21(2):125-30.

16. Miller N, Noble E, Jones D, Burn D. Life with communication changes in Parkinson's disease. Age and Ageing. 2006; 35: 235-9.

17. Menezes LN, Vicente LCC. Envelhecimento vocal em idosos institucionalizados. Rev. CEFAC. jan-mar, 2007; 9(1): 90-8.

18. Peña ME, Garcia RJ, Olalla JMD, Llanos EV, Miguel AG, Cordeiro XF. Impact of the most frequent chronic health conditions on the quality of life among 
people aged $>15$ years in Madrid. Eur $\mathrm{J}$ of Public Health. July, 2009; 20(1): 78-84.

19. Morzaria S, Damrose E. A comparison of the $\mathrm{VHI}, \mathrm{VHI}-10$, and $\mathrm{V}-\mathrm{RQOL}$ for measuring the effect of Botox therapy in adduction spasmodic dysphonia. J. Voice. in press, 2010.

20. Kummer A, Teixeira AL. Neuropsychiatry of Parkinson's disease. Arq Neuropsiquiatr. 2009; 67(3-B):930-9.

21. Nakabayashi TIK, Chagas MHN, Corrêa ACL, Tumas V, Loureiro SR, Crippa JAS. Prevalência de depressão na doença de Parkinson. Rev Psiq Clín. 2008;35(6):219-27.

22. Behlau M. Voz: o livro do especialista. Volume 1. Rio de Janeiro. Revinter, 2001.

23. Jones SM, Carding PN, Drinnan MJ. Exploring the relationship between severity of dysphonia and voice-related quality of life. Clin Otolaryngol. 2006; 31: 411-7.
24. Lana RC, Álvares LMRS, Nasciutti-Prudente C, Goulart FRP, Teixeira-Salmela LF, Cardoso FE. Percepção da qualidade de vida de indivíduos com doença de Parkinson através do PDQ-39. Rer Bras Fisioter. Set/out, 2007; 11(5):397-402.

25. Gama ACC, Menezes LM, Maia AA, Rezende Neto AL, Oliveira JB. Voice related quality of life after botulinum toxin injection for spasmodic dysphonia. Rev Laryngol Otol Rhinol. 2010; 131(1):39-44.

26. Azevedo LL, Cardoso F. Ação da levodopa e sua influência na voz e na fala de indivíduos com doença de Parkinson. Rev Soc Bras. Fonoaudiol. 2009; 14(1): 136-41.

27. Assencio-Ferreira VJ. Editorial II: a fonoaudiologia e a neurociência. Rev. CEFAC [online]. 2009; 11(3)
http://dx.doi.org/10.1590/S1516-18462012005000072

RECEBIDO EM: 03/10/2011

ACEITO EM: 11/12/2011

Endereço para correpondência:

Bárbara Pereira Lopes

Universidade Federal de Minas Gerais

Av. Alfredo Balena, 190 - sala 251

Belo Horizonte - MG

CEP: 30130-100

E-mail: babipereiralopes@gmail.com 